\title{
FIXED POINTS OF ENDOMORPHISMS OF COMPACT GROUPS
}

\author{
BY ROBERT F. BROWN
}

Communicated by Glen E. Bredon, September 22, 1973

1. Introduction. Let $G$ be a compact, connected Lie group and denote its real Čech cohomology by $H^{*}(G)$. Then $H^{*}(G)$ is an exterior algebra with generators $1=z_{0}, z_{1}, z_{2}, \cdots, z_{\lambda}$; where, by a theorem of Hopf [3], $\lambda$ is equal to the rank of $G$ (the dimension of a maximal torus). This paper announces some improvements of Hopf's result. The details will be published elsewhere.

2. Fixed point groups. For a set $X$ and a function $f: X \rightarrow X$, let $\Phi(f)$ denote the set of fixed points of $f$ : those $x \in X$ for which $f(x)=x$. If $X$ is a topological group and $f$ is a homomorphism, we will use the symbol $\Phi_{0}(f)$ for the component of the group $\Phi(f)$ which contains the identity element of $X$.

We consider a compact, connected Lie group $G$ and let $h$ be an automorphism of $G$. Choose algebra generators $1=z_{0}, z_{1}, z_{2}, \cdots, z_{\lambda}$ for $H^{*}(G)$ and let $\boldsymbol{H}^{*}(G)$ denote the linear span of $z_{1}, z_{2}, \cdots, z_{\lambda}$. The automorphism $h^{*}$ of $H^{*}(G)$ induced by $h$ takes $\boldsymbol{H}^{*}(G)$ to itself; let $\boldsymbol{h}^{*}$ denote the restriction of $h^{*}$ to $\boldsymbol{H}^{*}(G)$.

Our main result is

THEOREM 1. Let $G$ be a compact, connected Lie group and let $h$ be an automorphism of $G$. Then the rank of the Lie group $\Phi_{0}(h)$ is equal to the dimension of the vector space $\Phi\left(\boldsymbol{h}^{*}\right)$.

Note that Theorem 1 reduces to Hopf's theorem when $h$ is the identity function.

One might suspect that Theorem 1 is a consequence of some more intimate relationship between $H^{*}\left(\Phi_{c}(h)\right)$ and $\Phi\left(h^{*}\right)$. However, let $g \in G$ be a regular element and define $h(x)=g^{-1} x g$, for $x \in G$, then $h$ induces the identity isomorphism in cohomology, so $\Phi\left(h^{*}\right)=H^{*}(G)$; while $\Phi_{0}(h)$ is a maximal torus of $G$. Thus the possibilities for such a relationship are very limited.

AMS (MOS) subject classifications (1970). Primary 57F10; Secondary 22B05, 22C05, 22D35, 22E15, 22E99. 
The proof of Theorem 1 can be reduced to two special cases: when $G$ is abelian and when $G$ is semisimple.

In the abelian case we can work in a more general setting. Let $G$ be a compact, connected abelian topological group and let $h$ be an endomorphism of $G$. Denote the character group of $G$ by $G^{\wedge}$ and write the endomorphism of $G^{\wedge}$ induced by $h$ as $h^{\wedge}$. Using the techniques of Pontryagin duality theory, we prove

THEOREM 2. Let $h$ be an endomorphism of a compact, connected abelian topological group $G$, then the dimension of the group $\Phi_{0}(h)$ is equal to the torsion-free rank of $\Phi\left(h^{\wedge}\right)$.

Let $h^{*, 1}$ be the endomorphism of $H^{1}(G)$ induced by $h$. Representation theory and the continuity of Cech cohomology are used to prove

THEOREM 3. Let $h$ be an endomorphism of a compact, connected abelian topological group $G$, then the torsion-free rank of $\Phi\left(h^{\wedge}\right)$ is equal to the dimension of the vector space $\Phi\left(h^{*, 1}\right)$.

Theorems 2 and 3 imply Theorem 1 in the case that $G$ is abelian.

If $h$ is an automorphism of a compact, connected semisimple Lie group $G$, then $h^{m}$ ( $h$ composed with itself $m$ times) is an inner automorphism, for some $m \geqq 1$. We may assume that $h^{m}$ is, in fact, the identity automorphism. Let $\Gamma$ be the semidirect product, of $G$ and the cyclic group of order $m$, induced by $h$. Then there is an element $\gamma \in \Gamma$ such that $h(x)=\gamma^{-1} x \gamma$ for all $x \in \Gamma_{0}=G$. The proof of Theorem 1 , in the case that $G$ is semisimple, now follows from Theorem 4.3 of [1].

3. A bound on the rank. Let $\mathfrak{A}$ be a simple Lie algebra and define $\rho(\mathfrak{A})$ to be the minimum rank of $\Phi(\eta)$, for all automorphisms $\eta$ of $\mathfrak{A}$. The numbers $\rho(\mathfrak{H})$ are easily computed using Theorem 1 and material from [2].

THEOREM 4. Let $G$ be a compact, connected Lie group with Lie algebra (5. Write

$$
\mathfrak{G} \cong 3 \oplus \mathfrak{I}_{1}^{1} \oplus \cdots \oplus \mathfrak{I}_{1}^{k(1)} \oplus \cdots \oplus \mathfrak{A}_{u}^{1} \oplus \cdots \oplus \mathfrak{A}_{u}^{k(u)}
$$

where 3 is abelian, $\mathfrak{A}_{s}^{i} \cong \mathfrak{H}_{s}^{j} \cong \mathfrak{A}_{s}$ for each $s=1,2, \cdots, u$ and all $i$, $j=1, \cdots, k(s)$, and $\mathfrak{A}_{s}^{i} \nsubseteq \mathfrak{U}_{t}^{j}$ if $s \neq t$. Then

$$
\sum_{s=1}^{u} \rho\left(\mathfrak{U}_{s}\right) \leqq \operatorname{rank} \Phi_{0}(h)
$$

for all automorphisms $h$ of $G$. 
If, for example, $G$ is simply-connected, then there is an automorphism $h$ of $G$ such that rank $\Phi_{0}(h)$ is precisely $\sum_{s=1}^{u} \rho\left(\mathfrak{2}_{s}\right)$; so Theorem 4 cannot be improved in general.

COROLlary 4.1 (DE Siebenthal [4]). If $G$ is a compact, connected Lie group and there is an automorphism of $G$ with a finite set of fixed points, then $G$ is abelian.

COROLLARY 4.2. If there is an automorphism h of a compact, connected Lie group $G$ such that $\Phi_{0}(h)$ is a sphere, then either $G$ is abelian or its Lie algebra $\mathfrak{G}$ is of the form $\mathfrak{b} \cong \mathfrak{Z} \oplus \mathfrak{U} \oplus \cdots \oplus \mathfrak{U}$ where 3 is abelian and $\mathfrak{A}$ is a simple Lie algebra, either of type $A_{1}$ or of type $A_{2}$.

4. The power map. Let $G$ be a Lie group whose components are compact. In other words, $G$ is an extension of a compact, connected Lie group $G_{0}$ by a discrete, but not necessarily finite, group. Define the rank of a component $C$ of $G$ to be the rank of the identity component of the centralizer of $g$ in $G$, for any $g \in C$. Theorem 1 implies that the definition is independent of the choice of $g \in C$.

Define, for $k \geqq 2$, the "power map" $p_{k}: G \rightarrow G$ by $p_{k}(g)=g^{k}$. The component of $G$ containing an element $g$ is $g G_{0}$, so $p_{k}\left(g G_{0}\right) \subseteq g^{k} G_{0}$.

THEOREM 5. Let $G$ be a Lie group with compact components. The following are equivalent:

(i) $p_{k}\left(g G_{0}\right)=g^{k} G_{0}$

(ii) the degree of the map $p_{k}: g G_{0} \rightarrow g^{k} G_{0}$ is not zero,

(iii) $\operatorname{rank}\left(g G_{0}\right)=\operatorname{rank}\left(g^{k} G_{0}\right)$.

Theorem 5 extends the main result, Theorem 5.2, of [1]-for compact Lie groups-to Lie groups with compact components since, when $G$ is compact, the definition of the rank of a component given above agrees with the definition used in [1].

The equivalence of (ii) and (iii) follows easily from Theorem 1 above and Theorem 2.3 of [1]. Of course (ii) implies (i). The rest of the proofif the degree of $p_{k}: g G_{0} \rightarrow g^{k} G_{0}$ is zero then the dimension of $p_{k}\left(g G_{0}\right)$ is smaller than the dimension of $G$-reduces to the usual cases: $G_{0}$ abelian and $G_{0}$ semisimple. Following a suggestion of K. H. Hofmann, we consider the map $\varphi_{k}^{g}: G_{0} \rightarrow G_{0}$ defined by $\varphi_{k}^{g}(x)=g^{-k}(g x)^{k}$ and prove the equivalent statement: if the degree of $\varphi_{k}^{g}$ is zero, then $\varphi_{k}^{g}\left(G_{0}\right)$ has smaller dimension than $G_{0}$. In case $G_{0}$ is abelian, we again use Pontryagin duality theory. When $G_{0}$ is semisimple we can assume that $g^{m}$ is in the centralizer of $G_{0}$, for some $m \geqq 1$. This permits us to construct a Lie group with identity component $G_{0}$ and only $m$ components, apply Theorem 5.2 of [1] to 
that compact group, and then "lift" that information back to $G$ to obtain the desired result.

The author thanks V. S. Varadarajan for several helpful conversations concerning the material of this announcement.

\section{REFERENCES}

1. R. Brown, On the power map in compact groups, Quart. J. Math. Oxford Ser. (2) 22 (1971), 395-400. MR 46 \#298.

2. - The real cohomology of compact disconnected Lie groups, Proc. Amer. Math. Soc. 37 (1973), 255-259.

3. H. Hopf, Über den Rang geschlossener Liescher Gruppen, Comment. Math. Helv. 13 (1940), 119-143. MR 3, 35.

4. J. de Siebenthal, Sur les groupes de Lie compactes non connexes, Comment. Math. Helv. 31 (1956), 41-89. MR 20 \#926.

Department of Mathematics, University of California, Los Angeles, CaliFORNIA 90024 